\title{
Composition chimique et activité antibactérienne des huiles essentielles de Rosmarinus officinalis $L$. de la région de Hammamet (Tébessa-Algérie)
}

Manuscrit reçu le 24 août 2016 et accepté le 29 août 2016

\author{
Lamia BoutABIA $^{* 1,2}$, Salah Telailia ${ }^{1,2}$, Ismail Bouguetof ${ }^{1}$, \\ Faouzi GUENADIL ${ }^{1}$ et Azzedine CHEFrour ${ }^{3,4}$
}

${ }^{1}$ Université Chadli BENDJEDID El Tarf, 36000 El Tarf, Algérie

${ }^{2}$ Laboratoire Agriculture et Fonctionnement des Ecosystèmes, Faculté des Sciences de la Nature et de la Vie, Université Chadli BENDJEDID El Tarf, 36000 El Tarf, Algérie

${ }^{3}$ Université Mohamed Cherif MESSAADIA Souk Ahras, 41000 Souk Ahras, Algérie

${ }^{4}$ Laboratoire Développement et Contrôle des Préparations Pharmaceutiques Hospitalières, Faculté de Médecine, Université BADJI Mokhtar Annaba, 23000 Annaba, Algérie

\section{Résumé}

Le romarin (Rosmarinus officinalis) est une espèce très abondante dans la région de Hammamet de la wilaya de Tébessa à l'Est algérien, il est utilisé en médecine populaire, cosmétique et phytopharmacie.

L'extraction des huiles essentielles du romarin a été effectuée par entraînement à la vapeur d'eau. L'étude chimique de ces huiles essentielles par CPG-SM a révélé la richesse de ces dernières en 1,8 cinéole par rapport aux autres composés.

L'activité des huiles essentielles de Rosmarinus officinalis sur trois souches bactériennes pathogènes (Staphylococcus aureus, Escherichia coli, Acinetobacter sp.) par la technique d'aromatogramme montre que le pouvoir antimicrobien de ces huiles est très important et se caractérise par une action bactéricide contre les germes suscités.

Mots clés : Rosmarinus officinalis, huiles essentielles, pouvoir antimicrobien, aromatogramme, 1,8 cinéole, Tébessa, Algérie.

\begin{abstract}
Rosemary (Rosmarinus officinalis) is a very abundant species in the area of Hammamet at wilaya of Tébessa at eastern Algeria; it is used in folk medicine, cosmetics and phytopharmacy.

The extraction of the essential oil from rosemary was carried out by stripping with steam. Chemical study of these essential oils by GC-MS revealed the richness of the past in 1,8 cinéole compared to other compounds.

The activity of the essential oil of Rosmarinus officinalis on three pathogenic bacterial strains (Staphylococcus aureus, Escherichia coli, Acinetobacter sp.) by the technique of aromatogramme shows that the antimicrobial potency of this oil is very important and is characterized by bactericidal raised against germs.
\end{abstract}

Keywords: Rosmarinus officinalis, essential oil, antimicrobial power, aromatogramme, 1,8 cineol, Tébessa, Algeria.






\section{Introduction}

Pour mieux connaitre les vertus thérapeutiques d'une plante, il est nécessaire d'analyser la composition chimique de ses principes actifs qui nous permettra de lutter contre les infections produites par les bactéries ou certains autres agents pathogènes (Lehmann, 2013).

Les bactéries sont responsables de plusieurs maladies. Leur résistance aux antibiotiques est de plus en plus prononcée. Pour arrêter ce processus de synthèse-résistance, il est nécessaire de chercher une autre approche afin de diminuer ou d'éliminer les affections sans l'utilisation des produits synthétiques, donc il est évident de trouver des solutions par l'utilisation des molécules bioactives qui sont à base de plantes (Vanden Berghe et Vlietinck, 1991).

La situation est davantage plus préoccupante à cause de l'apparition des souches de microorganismes antibiorésistants et l'émergence des infections non communes qui compromettent les traitements à l'aide des médicaments existants. Face à ces nombreux obstacles que présente l'utilisation des antibactériens disponibles, il est indispensable de chercher de nouvelles substances antibactériennes efficaces et à large spectre d'action (Biyiti et al., 2004). L’Algérie est un pays très riche en plantes médicinales qui poussent généralement à l'état spontané (Baba Aïssa, 1991). La région de Hammamet de la wilaya de Tébessa est l’une des régions du pays connue pour sa richesse en plantes médicinales, aromatiques et mellifères, favorisée par sa position géographique et son climat semi-aride (Benarfa, 2005), mais qui reste encore méconnue par les uns et les autres. Pour ces raisons, nous avons effectué une étude sur l'une de ces plantes qui est le romarin (Rosmarinus officinalis). Cette plante est souvent utilisée efficacement par les tradipraticiens dans le traitement de diverses maladies infectieuses (Beloued, 1998) ainsi, notre étude porte sur la composition chimique des huiles essentielles et l'effet antibactérien par le biais d'un test d'aromatogramme.

\section{Matériel et méthodes}

\subsection{Matériel végétal}

Les parties aériennes de Rosmarinus officinalis ont été collectées en Mars 2014 de trois sites (Youkous, Draa Hammam et Ammacha) de la région de Hammamet, daïra de Bir M'Kadem, wilaya de Tébessa. La dite région appartient aux domaines des hautes plaines de l’Est algérien aux confins algéro-tunisien plus précisément sur le piémont des Nemamcha avec une superficie de $375 \mathrm{Km}^{2}$. Caractérisée par un climat semi-aride, ses coordonnées Lambert sont: $35^{\circ} 25^{\prime} \mathrm{N}$ et $7^{\circ} 55^{\prime} \mathrm{E}$. Son altitude est de $854 \mathrm{~m}$ (Benarfa, 2005). 
La matière végétale, recueillie, a été séchée à l'air libre, à l'ombre jusqu’à la stabilisation de son poids (= 7 jours).

\subsection{Méthodes}

\subsubsection{Extraction des huiles essentielles}

L'extraction des huiles essentielles a été effectuée par hydrodistillation durant une heure, à la température de $100^{\circ} \mathrm{C}$, des parties aériennes séchées de la plante dans un appareil de type Clevenger. La vapeur d'eau enrichie de constituants volatils est condensée puis décantée à $20^{\circ} \mathrm{C}$. Cette opération est suivie par le calcul des rendements effectué selon la norme AFNOR (1986).

\subsubsection{Analyse des huiles essentielles}

Les huiles essentielles obtenues ont été analysées par chromatographie en phase gazeuse couplée à la spectrométrie de masse (CPG de type PERKIN Elmer/SM de type Turbo masse). L’huile essentielle est diluée par de l'éthanol destiné à l'analyse chromatographique à 10\%. La température de l'appareil a été programmée à $60^{\circ} \mathrm{C}$ pendant 2 minutes, puis elle montera crescendo (à raison de $3^{\circ} \mathrm{C}$ par minute) jusqu'à $200^{\circ} \mathrm{C}$ où elle se stabilisera pendant 4 minutes. Le balayage se fait pendant une heure pour avoir tous les composés. L’injection se fait, quand la température de $200^{\circ} \mathrm{C}$ est atteinte, par un mode «SPLIT» à raison de $0,3 \mu$ l d'huile essentielle diluée.

L'identification des composants a été effectuée par comparaison entre les données spectrales et chromatographiques et celles de la littérature (Adams, 1995).

\subsubsection{Méthode d'évaluation de l'activité antibactérienne}

Choix des souches

Le choix des souches testées en fonction de l'utilisation en médecine traditionnelle du romarin; on a choisis trois souches: deux de gram négatif: Escherichia coli (pour les infections digestifs) et Acinetobacter sp. (la flore commensale de la peau chez l'homme) et une de gram positif Staphylococcus aureus (pour les infections nosocomiales).

\section{Méthode de l'aromatogramme}

L'activité antibactérienne est évaluée par la méthode d'aromatogramme qui permet de déterminer la sensibilité des différentes espèces bactériennes vis à vis de l’huile essentielle donnée. La méthode de l'aromatogramme consiste à utiliser des boites de Pétri contenant un 
milieu gélosé convenable, déjà solidifié et inoculé de la souche microbienne testée. Des disques en papier buvard de $6 \mathrm{~mm}$ de diamètre, préalablement imprégnés de quantités connues d’huile essentielle $(07 \mu 1)$, sont alors placés en surface de la gélose. Généralement, les microorganismes seront classés susceptibles, intermédiaires ou résistants, selon le diamètre de la zone d'inhibition (Wilkinson, 2006).

\section{Mesure de l'activité antimicrobienne des huiles essentielles}

La technique de l'aromatogramme a été appliquée dans le but d'évaluer l'activité antimicrobienne des huiles essentielles. Ce test est effectué par dépôt d'un disque stérile de 6 mm de diamètre imprégné d'une quantité d’huile essentielle sur un milieu gélosé préalablement ensemencé avec une culture microbienne. Après incubation, la lecture des résultats se fait par mesure des diamètres des zones d'inhibition en millimètres (Chao et al.,2000; Andrews, 2001).

Après ensemencement des germes, trois dilutions H.E (huiles essentielles) (concentrée, 1/2 et 1/4) sont préparées à l'aide du DMSO (diméthylsulfoxyde) qui est inerte sur l'activité bactérienne.

Dans chaque boite de Pétri ensemencée on applique 5 disques:

* Le premier est un disque Témoin sans huile essentielle et sans DMSO.

* Le deuxième imbibé par le DMSO pour confirmer la non activité sur les germes.

* Le troisième imprégné par l’huile essentielle pure.

* Le quatrième disque imbibé par l’huile essentielle diluée à 1⁄2 par le DMSO.

* Le cinquième est imbibé par l’huile essentielle diluée à 1/4 par le DMSO.

\section{Détermination de la sensibilité}

La sensibilité aux différentes huiles essentielles est organisée selon le diamètre des zones d'inhibition comme suit: non sensible (-) pour le diamètre moins sensible de $6 \mathrm{~mm}$; sensible $(+)$ pour un diamètre entre 9-14 $\mathrm{mm}$; très sensible $(++)$ pour un diamètre entre $15-19 \mathrm{~mm}$ et extrêmement sensible (+++) pour le diamètre plus que $20 \mathrm{~mm}$ (Ponce et al.,2003).

Les souches ont été testées par les antibiotiques indiqués dans le tableau 1. 
Bulletin de la Société Royale des Sciences de Liège, Vol. 85, 2016, p. 174 - 189

Tableau 1 : Liste des antibiotiques testés pour les souches bactériennes utilisées

\begin{tabular}{ccc}
\hline Staphylococcus sp. & Escherichia sp. & Acinetobacter sp. \\
\hline \hline Oxacilline (OX) & Amoxicilline + acide & Pipéracilline(PIP) \\
clavulanique (AUG) & \\
Vancomycine (VA) & Cefotaxime (CTX) & Amikacine(AK) \\
Trimethoprime / & Tobramycine(TOB) \\
Sulfamethoxazole (SXT) & Ampicilline (AMP) & Trimethoprime / \\
& Trimethoprime / & \\
(K) & Sulfamethoxazole (SXT) & (CAZ) \\
Tétracycline (TE) & Gentamicine (CN) & Imipenème (IMI) \\
Gentamicine (CN) & Amikacine (AK) & Colistine (CS) \\
Erythromycine (E) & Cefazoline (KZ) & Tétracycline (TE) \\
(L) & (MA) & Pefloxacine (PEF) \\
(FA) & Tétracycline (TE) & Rifampicine (RD) \\
Chloramphénicol (C) & Chloramphénicol (C) & Gentamicine (CN) \\
Amikacine (AK) & (NOR) & Aztréonam(ATM) \\
Pefloxacine (PEF) & Colistine (CS) & \\
Rifampicine (RD) & Pefloxacine (PEF) & \\
& Ceftriaxone (CRO) & \\
& Imipenème (IMI) & \\
& & \\
& &
\end{tabular}

\section{Résultats}

\subsection{Rendement}

L'opération d'extraction des huiles essentielles du romarin dans les trois stations de la région de Hammamet a permis d'avoir des rendements allant de $1.60 \pm 0.004 \mathrm{ml} / 100 \mathrm{~g}$ MS à $2.29 \pm$ $0.041 \mathrm{ml} / 100 \mathrm{~g}$ MS. Le tableau 2 regroupe les rendements obtenus de chaque station d'étude.

Tableau 2 : Rendement en huile essentielle du romarin des stations d'étude

\begin{tabular}{lcccccc}
\hline \multicolumn{1}{c}{ Station } & RHE1 & RHE2 & RHE2 & RHE 4 & $\begin{array}{c}\text { Moyenne } \\
\text { ml/100gMS }\end{array}$ & Écart type \\
\hline \hline & & & & & & \\
Station 1 (Youkous) & 2.25 & 2.27 & 2.36 & 2.30 & 2.29 & 0.041 \\
Station 2 (Draa Hammam) & 1.60 & 1.57 & 1.63 & 1.61 & 1.60 & 0.004 \\
Station 3 (Ammacha) & 1.83 & 1.96 & 1.77 & 1.84 & 1.85 & 0.004 \\
\hline
\end{tabular}


Bulletin de la Société Royale des Sciences de Liège, Vol. 85, 2016, p. 174 - 189

\subsection{Composition chimique de l'huile essentielle du romarin des trois stations étudiées}

La composition chimique des huiles essentielles du romarin des stations étudiées de la région de Hammamet est consignée dans le tableau 3.

Tableau 3: Composition chimique des huiles essentielles du romarin des stations d'étude

\begin{tabular}{|c|c|c|c|c|c|}
\hline & Localité & & Youkous & Draa Hammam & Ammacha \\
\hline $\mathrm{N}^{\circ}$ & Composés & $\overline{\mathrm{IR}}$ & $\begin{array}{c}\text { Pourcentage } \\
(\%)\end{array}$ & $\begin{array}{c}\text { Pourcentage } \\
\text { (\%) }\end{array}$ & $\begin{array}{c}\text { Pourcentage } \\
(\%)\end{array}$ \\
\hline 1 & $\alpha$-Pinène & 932 & - & 3.39 & 3.41 \\
\hline 2 & Camphène & 949 & - & 14.40 & 14.47 \\
\hline 3 & Sabinène & 980 & 2.40 & 15.93 & 16.01 \\
\hline 4 & $\beta$-Pinène & 987 & - & - & 1.47 \\
\hline 5 & p-Cymène & 993 & - & 1.58 & 1.59 \\
\hline 6 & 1,8 cinéole & 1032 & 72.91 & 32.59 & 32.76 \\
\hline 7 & $\gamma$-Terpinène & 1047 & - & 1.74 & - \\
\hline 8 & $\alpha$-Terpinolène & 1088 & 0.50 & 1.19 & 1.19 \\
\hline 9 & Linalol & 1094 & - & 0.10 & 0.10 \\
\hline 10 & Camphre & 1144 & 17.16 & 4.46 & 4.48 \\
\hline 11 & Pinocamphone & 1154 & - & 6.17 & 6.20 \\
\hline 12 & Bornéol & 1169 & 4.18 & 9.68 & 9.73 \\
\hline 13 & Terpinène-4-ol & 1178 & 1.88 & 3.64 & 3.66 \\
\hline 14 & 1-Dodecène & 1183 & - & 0.09 & 0.09 \\
\hline 15 & 1- $\alpha$-Terpineol & 1188 & 0.96 & 3.31 & 3.32 \\
\hline 16 & Verbénone & 1228 & - & 1.02 & 1.02 \\
\hline 17 & NI & 1229 & - & 0.15 & 0.15 \\
\hline 18 & NI & 1253 & - & 0.11 & - \\
\hline 19 & Bornyl acétate & 1287 & 0.01 & 0.30 & 0.31 \\
\hline 20 & $\alpha$-Copaène & 1378 & - & 0.04 & 0.04 \\
\hline 21 & Tetradecène & 1386 & - & 0.12 & - \\
\hline
\end{tabular}

IR: indice de rétention (min) ; NI : non identifié 
Comme le montre le tableau 3, les huiles essentielles obtenues n’ont pas la même composition. On note une différence de composition relativement importante notamment des huiles essentielles extraites de Rosmarinus officinalis de Draa Hammam et Ammacha par rapport à celles de Youkous. Les résultats obtenus par l'analyse chromatographique (CPG/SM) du romarin procuré des stations d'étude de la région de Hammamet indiquent que l'huile essentielle de l'échantillon de Draa Hammam contient 20 composants chimiques; celle de l'échantillon de Ammache comprend 18 composants et celle de Youkous englobe uniquement 8 composants. Par ailleurs, il est important de noter que le chémotype à pourcentage élevé des huiles essentielles étudiées est le 1,8 cinéole avec respectivement: 72,91\% échantillon de Youkous; 32,59\% échantillon de Draa Hammam et 32.76\% échantillon de Ammacha.

\subsection{Activité antibactérienne de l'huile essentielle du romarin}

\subsubsection{Résultats de l'aromatogramme}

Le teste d'aromatogramme de l'huile essentielle du romarin des stations étudiées au niveau de la région de Hammamet de la wilaya de Tébessa est consignée dans le tableau 4. 
Bulletin de la Société Royale des Sciences de Liège, Vol. 85, 2016, p. 174 - 189

Tableau 4: Diamètres d'inhibition (mm) des différentes souches testées dans l'aromatogramme en milieu solide

\begin{tabular}{|c|c|c|c|c|c|c|c|c|c|c|c|c|c|c|c|c|c|c|}
\hline \multirow{2}{*}{$\begin{array}{l}\text { Souche } \\
\text { Dilution }\end{array}$} & \multicolumn{6}{|c|}{ Youkous } & \multicolumn{6}{|c|}{ Draa Hammam } & \multicolumn{6}{|c|}{ Ammacha } \\
\hline & $\begin{array}{c}\text { S. aureus } \\
\text { (25923) }\end{array}$ & $\begin{array}{l}\text { S. aureus } \\
\text { (SAMR) }\end{array}$ & $\begin{array}{c}\text { E. coli } \\
\text { (sensible) }\end{array}$ & $\begin{array}{l}\text { E. coli } \\
\text { (BLSE) }\end{array}$ & $\begin{array}{c}\text { Ac. sp } \\
\text { Sensible }\end{array}$ & $\begin{array}{l}\text { Ac. sp } \\
\text { (BMR) }\end{array}$ & $\begin{array}{c}\text { S. aureus } \\
\text { (25923) }\end{array}$ & $\begin{array}{l}\text { S. aureus } \\
\text { (SAMR) }\end{array}$ & $\begin{array}{c}\text { E. coli } \\
\text { (sensible) }\end{array}$ & $\begin{array}{c}\text { E. coli } \\
\text { (BLSE) }\end{array}$ & $\begin{array}{c}\text { Ac. sp } \\
\text { Sensible }\end{array}$ & $\begin{array}{l}\text { Ac. sp } \\
\text { (BMR) }\end{array}$ & $\begin{array}{c}\text { S. aureus } \\
\text { (25923) }\end{array}$ & $\begin{array}{l}\text { S. aureus } \\
\text { (SAMR) }\end{array}$ & $\begin{array}{c}\text { E. coli } \\
\text { (sensible) }\end{array}$ & $\begin{array}{l}\text { E. coli } \\
\text { (BLSE) }\end{array}$ & $\begin{array}{c}\text { Ac. sp } \\
\text { Sensible }\end{array}$ & $\begin{array}{r}\text { Ac. sp } \\
\text { (BMR) }\end{array}$ \\
\hline Disque seul & 6 & 6 & 6 & 6 & 6 & 6 & 6 & 6 & 6 & 6 & 6 & 6 & 6 & 6 & 6 & 6 & 6 & 6 \\
\hline $\begin{array}{l}\text { Disque } \\
\text { DMSO }\end{array}$ & 6 & 6 & 6 & 6 & 6 & 6 & 6 & 6 & 6 & 6 & 6 & 6 & 6 & 6 & 6 & 6 & 6 & 6 \\
\hline Huile pure & 17.1 & 16.4 & 17.2 & 15.6 & 14 & 15 & 18.1 & 16.1 & 16.8 & 14.7 & 13 & 13 & 15.3 & 15.8 & 16.2 & 14.8 & 13 & 13 \\
\hline Dilution 1/2 & 10.1 & 9.1 & 8.1 & 6 & 8.9 & 7.2 & 11.2 & 10.6 & 9.5 & 6 & 8.88 & 7.1 & 11.1 & 9.8 & 9.1 & 7 & 10 & 8.2 \\
\hline Dilution $1 / 4$ & 8.2 & 7.2 & 7.3 & 6 & 10 & 7.2 & 9.2 & 6.2 & 7.5 & 6 & 10.1 & 7.4 & 7.2 & 6.2 & 7.8 & 6 & 10 & 7 \\
\hline
\end{tabular}


À l'issue des résultats obtenus à travers le tableau 4, il ressort que les bactéries examinées sont très sensibles à l'huile pure. Par contre, ces germes ont une sensibilité relative vis-à-vis des dilutions $1 / 2$ et/ou $1 / 4$ des huiles essentielles du romarin récolté des stations suscitées.

\section{Discussion}

Les rendements des huiles essentielles obtenues à partir des trois populations étudiées au niveau de la région de Hammamet de la wilaya de Tébessa sont conformes aux normes d'AFNOR. Cependant, ils montrent une différence significative entre l'échantillon de Youkous (2.29 ml/100g MS) et les échantillons de Draa Hammam et Ammacha (1.60ml/100g MS à 1.85 ml/100g MS). Par ailleurs, la moyenne des rendements obtenus (1.91 ml/100g MS) est supérieure à celle trouvée par Baghloul (2007) à partir d’un échantillon récolté au mois de Juin 2006 de la région de Hammamet (Tébessa) soit un rendement de 1.69 ml/100g MS. Ce qui explique que la période de floraison (Mars) serait propice pour la collecte de ce genre de plante aromatique, car ses principes actifs sont synthétisés et stockés dans les feuilles et les sommités fleuries (Boutekedjiret et al., 1999).

L'analyse des huiles essentielles du romarin de la région de Hammamet s'avère très intéressante, elle nous a permis de constater que le nombre des composants obtenu par analyse chromatographique (CPG/SM) est important soit 21 composants chimiques, ce qui se rapproche du nombre obtenu par Fournier et ses collaborateurs (1983) pour le romarin de Tunisie (frontière avec notre région d'étude) dont l'analyse de l'huile a abouti à l'identification de 26 constituants. Cependant dans leur étude relative à l'analyse des huiles essentielles du romarin de provenances géographiquement différentes d'Algérie (Alger, Bibans, Djelfa et Laghouat), Boutekedjiret et ses collaborateurs (2005) ont énuméré entre 28 et 61 composants chimiques. Par ailleurs, la composition chimique du romarin des stations étudiées de la région de Hammamet montre nettement que le 1,8 cinéole est le chémotype majoritaire des huiles essentielles. Ce qui diffère des résultats obtenus à travers plusieurs travaux qui ont mis en évidence l'existence de multiples chémotypes d'huile essentielle de Rosmarinus officinalis dans différentes régions d'Algérie. Nous citerons ceux de Lograda et ses collaborateurs (2013) qui ont noté que la composition chimique des huiles essentielles du romarin collecté de cinq régions de l'Est algérien est dominée par le camphre (42,7\%) et de Atik Bekkara et ses collaborateurs (2007) où l'huile essentielle du romarin spontané de la région de Tlemcen à l'Ouest algérien serait caractérisée par la présence de l' $\alpha$-Pinène (23,1\%). 
Cependant, l'étude menée par Boutekedjiret et ses collaborateurs (1998) indique que les huiles essentielles du romarin des Biban de la région de Bordj Bou Arreridj, sont dominées par un chémotype spécifique qu'est le 1,8 cinéole. Ce qui concorde avec notre résultat.

Les études effectuées sur la composition chimique des huiles essentielles du romarin en Tunisie, Maroc, Espagne et Italie, déterminent l'importance du chémotype 1,8-cinéol (Varela et al., 2009; Ayadi et al., 2011; Hcini et al., 2013; Jordán et al., 2013; Khia et al., 2014; Tahri et al., 2015; Tuttolomondo et al., 2015).

En France, l'huile essentielle du Rosmarinus officinalis de la Provence a un niveau élevé de camphre (30-45\%) (Kaloustian et al., 2002) par contre celle de la Corse serait caractérisée par l' $\alpha$-pinène (13.7-24.6\%) (Pintore et al., 2002).

Cette variabilité des résultats de l'huile essentielle de romarin peut être due à des facteurs intrinsèques (génétique, sous-espèces) ou à des facteurs extrinsèques comme le climat et le sol (origine géographique) ou à la méthode d'extraction (Özcan et Chalchat, 2008).

Les résultats obtenus à partir de l'aromatogramme ont montrés que les huiles essentielles pures du romarin de la région de Hammamet possède une activité antibactérienne contre les micro-organismes sélectionnés dans le cadre du protocole entrepris. Seulement, il faudrait mentionner que la sensibilité des souches testées varie selon les dilutions. Les Staphylococcus aureus (25923) et Staphylococcus aureus (SAMR), ont été les bactéries les plus sensibles aux extraits du Rosmarinus officinalis des trois provenances. Jordán et ses collaborateurs (2013) mentionnent qu'une forte proportion d' $\alpha$-pinène augmente l'efficacité des huiles essentielles du romarin contre Staphylococcus aureus, tandis que la présence de 1,8 cinéole, comme le composé le plus abondant, diminue considérablement l'efficacité de ces huiles. En revanche, l'efficacité des huiles pures contre Escherichia coli n'a pas été affectée par cette condition. Par ailleurs, le germe Escherichia coli (BLSE) représente toujours une résistance aux dilutions 1/2 et $1 / 4$ avec les huiles essentielles des trois provenances. La souche Acinetobacter sp. (sensible) représente une sensibilité dans la dilution $1 / 4$ que la dilution $1 / 2$ de Draa Hammam cela est dû peut être aux diffusions des composants de cette huile par une dilution élevée.

La susceptibilité des bactéries est en effet indépendante du gram (Dorman et Deans, 2000), ou dépendante des huiles essentielles utilisées (Deans et Ritchie, 1987). La sensibilité des microorganismes peut varier selon le germe testé car une huile essentielle peut être bactéricide visà-vis de certaines souches, bactériostatique vis-à-vis d'autres ou n'avoir aucun effet (Hermal, 1993). 
Le mécanisme d'action des huiles essentielles est lié essentiellement à la structure de la paroi et à la perméabilité membranaire des bactéries. L'huile essentielle exerce son pouvoir antimicrobien par son interférence avec la bicouche lipidique de la bactérie grâce à sa propriété hydrophobe, ce qui entraîne: l'augmentation de la perméabilité puis la perte des constituants cellulaire; l'acidification de l'intérieure de la bactérie, bloquant la production de l'énergie cellulaire et la synthèse des composants de structure; la destruction du matériel génétique, conduisant à la mort de la bactérie (Caillet et Lacroix, 2007). En revanche, les bactéries à Gram- sont plus résistantes que les Gram+, ceci est dû aux différences structurales de leurs membranes externes (Burt, 2004).

Par ailleurs, la sélectivité des huiles essentielles envers certaines bactéries restent encore mal élucidée. Cette sélectivité est le résultat de la composition variée des fractions actives des huiles, qui présentent souvent des actions synergiques (Hammer et al., 1999).

Des études antérieures sur l'huile essentielle du Rosmarinus officinalis révèlent une activité antimicrobienne et indiquent une similitude avec les résultats obtenus dans le présent travail (Moreira et al., 2005; Santoyo et al., 2005; de Billerbeck, 2007; Ouibrahim et al., 2013; Lograda et al., 2014; Belkhiri, 2015).

En raison de leur activité antimicrobienne, les huiles essentielles du romarin peuvent être utilisées dans l'industrie pharmaceutique pour la production de nouveaux agents synthétiques dans le traitement des maladies infectieuses causées par les bactéries étudiées ou peuvent être suggérées comme agents naturels de conservation des aliments comme l'a été rapporté par certains auteurs: Fernández-López et al. (2005), Balentine et al. (2006), Miladi et al. (2013), Tahri et al. (2015) et Hendel et al. (2016).

\section{Conclusion}

Le présent travail est consacré à la détermination du rendement, de la composition chimique et des propriétés antibactérienne des huiles essentielles extraites du Rosmarinus officinalis récolté dans la région de Hammamet de la wilaya de Tébessa en Mars 2014. Le rendement moyen en huile essentielle est de $1.91 \mathrm{ml} / 100 \mathrm{~g}$ MS. Les analyses chimiques, par CPG/SM, ont permis d'identifier 21 composants et indiquent que le chémotype le plus important serait le 1,8 cinéole. L'évaluation de l'activité antimicrobienne des huiles essentielles du romarin des stations d'étude a montré une efficacité contre les bactéries testés (Staphylococcus aureus, Escherichia coli, Acinetobacter sp.) et ce par la méthode de diffusion en milieu solide. 
Bulletin de la Société Royale des Sciences de Liège, Vol. 85, 2016, p. 174 - 189

Au regard des résultats de l'antibiogramme, il ressort que l'action des antibiotiques révèle des réponses variables pour les souches testées; soit des germes de Gram+ ou Gram-.

\section{Références}

Adams R.P., 1995- Identification of essential oil components by Gas chromatography/Mass spectroscopy. Allured Publisching Corporation. Carol Stream; Illinois, USA.

AFNOR, 1986- Recueil des Normes Françaises « huiles essentielles », AFNOR. Paris. 57 p.

Andrews J.M., 2001-The Development of the BSAC standardized method of disc diffusion testing. J. Antimic. Chemo., 48 (1): 29-42.

Atik Bekkara F., Bousmaha L., Taleb Bendiab S.A., Boti J.B. \& Casanova J., 2007Composition chimique de l'huile essentielle de Rosmarinus officinalis L. poussant à l'état spontané et cultivé de la région de Tlemcen. Biologie \& Santé, 7: 6-11.

Ayadi S., Jerribi C. \& Abderrabba M., 2011- Extraction et étude des huiles essentielles de Rosmarinus officinalis cueillie dans trois régions différentes de la Tunisie. J. Soc. Alger. Chim., 21 (1): 25-33.

Baba Aïssa F., 1991- Les plantes médicinales en Algérie. Bouchène et Ad. Diwan éd., Alger. $181 \mathrm{p}$.

Baghloul F., 2007- Caractérisation chimique et activité antimicrobienne de l'huile essentielle du Romarin. Mémoire Magister en Biochimie Appliquée. Univ. Badji Mokhtar-Annaba, 62 p.

Balentine C.W., Crandall P.G., O’Bryan C.A., Duong D.Q. \& Pohlman F.W., 2006- The pre- and post-grinding application of rosemary and its eVects on lipid oxidation and color during storage of ground beef. Meat Science,73: 413-421.

Belkhiri F.-Z., 2015- Etude de l'activités antibactérienne des huiles essentielles de Rosmarinus officinalis L. Master II Génie des Procédés, Univ. Mohamed Khider-Biskra. 51 p.

Beloued A., 1998- Plantes médicinales d'Algérie. Ed. office des publications universitaires. 277 p.

Benarfa N., 2005- Inventaire de la faune apoidienne dans la région de Tébessa. Mémoire Magister, Univ. Mentouri-Constantine. 130 p. 
Biyiti L., Meko'o D., Tamzc V. \& Amvam Zollo P., 2004- Recherche de l'activité antibactérienne de quatre plantes médicinales camerounaises. Pharm. Med. Trad. Afr., 13: 1120.

Boutekedjiret C., Buatois B. \& Bessiere J.M., 2005- Characterisation of Rosemary essential oil of different areas of Algeria. Journal of Essential Oil Bearing Plants, 8 (1): 65-70.

Boutekedjiret C., Bentahar F., Belabbes R. \& Bessiere J.M., 1998- The essential oil from Rosmarinus officinalis L. in Algeria. J. Essent. Oil Res., 10: 680-682.

Boutekedjiret C., Bentahar F., Belabbes R. \& Bessiere J.M., 1999- Study of Rosmarinus officinalis L. essential oil yield and composition as a function of the plant life cycle. J. Essent. Oil Res, 11 (2): 238-240.

Burt S., 2004- Essential oils: their antibacterial properties and potential applications in foods: a review. Int. J. Food Microbiol., 94: 223-253.

Caillet S. \& Lacroix M., 2007- Les huiles essentielles: leurs propriétés antimicrobiennes et leurs applications potentielles en alimentaire. INRS-Institut Armand-Frappier, RESALA, 1-8.

Chao S.C., Young D.G. \& Oberg G.J., 2000- Screening for Inhibitory Actvity of Essential Oils on Selected Bacteria, Fungi and Viruses. J. Essent. Oil Res., 12: 639-649.

de Billerbeck V.G., 2007- Huiles essentielles et bactéries résistantes aux antibiotiques. Phytothérapie, 5 (5): 249-253.

Deans S.G. \& Ritchie G., 1987- Antibacterial properties of plant essential oils. International Journal of Food Microbiology, 5 (2): 165-180.

Dorman H.J. \& Deans S.G., 2000- Antimicrobial agents from plants: antibacterial activity of plant volatile oils. Journal of Applied Microbiology, 88 (2): 308-16.

Fernández-López J., Zhi N., Aleson-Carbonell L., Pérez-Alvarez J.A. \& Kuri V., 2005Antioxidant and antibacterial activities of natural extracts: application in beef meatballs. Meat Science, 69: 371-380.

Fournier G., Habib J., Reguigui A., Safta F., Guetari S. \& Chemli R., 1983- Etudes de divers échantillons d’huiles essentielles de Romarin de Tunisie. Plant. Médicin. Phytothér., 23: 180-185.

Hammer K.A., Carson C.F. \& Riley T.V., 1999- Antimicrobial activity of essential oils and other plant extracts. J. Appl. Microbiology, 86: 985-990. 
Hcini K., Sotomayor J.A., Jordán M.J. \& Bouzid S., 2013- Chemical Composition of the Essential Oil of Rosemary (Rosmarinus officinalis L.) of Tunisian Origin. Asian Journal of Chemistry, 25 (5): 2601-2603.

Hendel N., Larous L. \& Belbey L., 2016- Antioxidant activity of rosemary (Rosmarinus officinalis L.) and its in vitro inhibitory effect on Penicillium digitatum. International Food Research Journal, 23(4): 1725-1732.

Hermal C., 1993- Activité bactériostatique de sept émulsions d'huiles essentielles et de deux associations d'émulsions d'huiles essentielles. Thèse, Faculté de pharmacie, Université Montpellier I. 87 p.

Jordán M.J., Lax V., Rota M.C. \& Sotomayor J.A., 2013- Effect of bioclimatic area on the essential oil composition and antibacterial activity of Rosmarinus officinalis L. Food Control, 30 (2): 463-468.

Kaloustian J., Portugal H., Pauli A.M. \& Pastor J., 2002- Chemical, Chromatographic and Thermal Analysis of Rosemary (Rosmarinus officinalis). J. Appl. Polym. Sci., 83: 747-756.

Khia A., Ghanmi M., Satrani B., Aafi A., Aberchane M., Quaboul B., Amusant N. \& Charrouf Z., 2014- Effet de la provenance sur la qualité chimique et microbiologique des huiles essentielles de Rosmarinus officinalis L. du Maroc. Phytothérapie, 12 (6): 341-347.

Lehmann H., 2013- Le médicament à base de plantes en Europe. Statut, Enregistrement, contrôle. Thèse pour l’obtention du grade de docteur de Strasbourg. Vol. I., 229 p.

Lograda T., Ramdani M., Chalard P. \& Figueredo G., 2013- Characteristics of essential oils of Rosmarinus officinalis from Eastern Algeria. Global J Res. Med. Plants \& Indigen. Med., 2 (12): 794-807.

Lograda T., Ramdani M., Chalard P. \& Figueredo G., 2014- Antibacterial activity of essential oils of Rosmarinus officinalis from Eastern Algeria. Global J Res. Med. Plants \& Indigen. Med., 3 (6): 232-242.

Miladi H., Ben Slama R., Mili D., Zouari S., Bakhrouf A. \& Ammar E., 2013- Essential oil of Thymus vulgaris L. and Rosmarinus officinalis L.: Gas chromatography-mass spectrometry analysis, cytotoxicity and antioxidant properties and antibacterial activities against foodborne pathogens. Natural Science, 5(6): 729-739. 
Moreira M.R., Ponce A.G., de Valle C.E. \& Roura S.I., 2005- Inhibitory parameters of essential oils to reduce a food borne pathogen. Lebensmittel-Wissenschaft und -TechnologieLWT, 38: 565-570.

Ouibrahim A., Tlili-Ait-Kaki Y., Bennadja S., Amrouni S., Djahoudi A.G. \& Djebar M.R., 2013- Evaluation of antibacterial activity of Laurus nobilis L., Rosmarinus officinalis L. and Ocimum basilicum L. from Northeast of Algeria. African Journal of Microbiology Research, 7 (42): 4968-4973.

Ozcan M.M. \& Chalchat J.C., 2008- Chemical composition and antifungal activity of rosemary (Rosmarinus officinalis L.) oil from Turkey. International J. Food Sci. Nutr., 59 (78): 691-698.

Pintore G., Usai M., Bradesi P., Juliano C., Boatto G., Tomi F., Chessa M., Cerri R. \& Casanova J., 2002- Chemical composition and antimicrobial activity of Rosmarinus officinalis L. oils from Sardinia and Corsica. Flavour and Fragrance Journal, 17: 15-19.

Ponce A.G., Fritz R., del Valle C.E. \& Roura S.I., 2003- Antimicrobial activity of essential oils on the native microfora of organic Swiss chard. Lebensmittel-Wissenschaft und Technologie, 36: 679-684.

Santoyo S., Cavero S., Jaime L., Ibañez E., Señoráns F.J. \& Reglero G., 2005- Chemical composition and antimicrobial activity of Rosmarinus officinalis L. essential oil obtained via supercritical fluid extraction. J. Food Prot., 68 (4): 790-795.

Tahri M., Imelouane B., Amhamdi H., Fauconnier M.-L. \& Elbachiri A., 2015- The Chemical compositions and the Antioxidant and Antimicrobial Activities of the Essential Oil of Rosemary Leaves from Eastern Morocco. J. Mater. Environ. Sci., 6 (3): 666-672.

Tuttolomondo T., Dugo G., Ruberto G., Leto C., Napoli E.M., Cicero N., Gervasi T., Virga G., Leone R., Licata M. \& La Bella S., 2015- Study of quantitative and qualitative variations in essential oils of Sicilian Rosmarinus officinalis L. Nat. Prod. Res., 29 (20):19281934.

Vanden Berghe D.A. \& Vlietinck, A.J., 1991- Screening for antibacterial and antiviralagents. In: Hostettmann, K. (Ed.), Methods in Plant Biochemistry, Vol. 6, Assays for Bioactivity. London, Academic Press, 47-59. 
Bulletin de la Société Royale des Sciences de Liège, Vol. 85, 2016, p. 174 - 189

Varela F., Navarrete P., Cristobal R., Fanlo M., Melereo R., Sotomayor J. A., Jordán M.J., Cabot P., Sanchez de Ron D., Calvo R. \& Cases A., 2009- Variability in the chemical composition of wild Rosmarinus officinalis L. Acta Horti., 826: 167-174.

Wilkinson J.M., 2006- Methods for testing the antimicrobial activity of extracts. Chapiter VIII.pp.157-165. In Ahmad I., Aqil F. and Owais M. Modern Phytomedicine : Turning Medicinal Plants into Drugs. Ed. WILEY-VCH Verlag GmbH \& Co. KGaA, Weinheim, 405 p. 